\title{
La place de la mathématique : Badiou avec Lacan'
}

\section{"La science sans conscience »'}

La mathématique peut-elle s'interroger sur elle-même ? Dans la Critique de la raison pure, Kant fait de la mathématique « l'exemple le plus éclatant d'une raison pure qui réussit à s'étendre d'elle-même sans le secours de l'expérience ${ }^{2}$ ». La définition kantienne de la mathématique résonne avec le formalisme de la mathématique moderne dont l'ascèse particulière exclut toute considération sur le contenu. Ainsi, la mathématique pure, selon Bertrand Russell, est « la classe de toutes les propositions de la forme 'P implique Q' ... où ni $\mathrm{P}$ ni $\mathrm{Q}$ ne contiennent d'autres constantes que des constantes logiques ${ }^{3}$ ». Selon définition russellienne, la mathématique pure se présente comme un ensemble d'implications formelles indépendantes de tout contenu. En forçant un peu le trait, on pourrait dire que la mathématique est « la science qui coïncide le plus totalement avec sa propre écriture 4 ». Mais pour le mathématicien d'aujourd'hui, l'enjeu de l'écriture mathématique ouvre une autre problématique, celle qui concerne le rapport du mathématicien avec la mathématique. Vu dans cette perspective, le mathématicien est

celui qui efface le plus totalement les marques de la production de son texte, tant les marques du sujet qui le produit que les marques de la société dans laquelle il est produit. C'est même en ce texte mathématique que l'effacement des marques est le plus explicite. Mais ce ne peut être là qu'un paradoxe, car le caractère ex-

1 Cet article est le résultat du programme de recherche P6-0014 «Conditions et problèmes de la philosophie contemporaine», financé par l’Agence slovène recherche.

2 Emmanuel Kant, Critique de la raison pure, Euvres philosophique, tome I : Des premiers écrits à la Critique de la raison pure (1747-1781), Bibliothèque de la Pléiade, Gallimard, Paris 1980, p. 1297.

3 Bertrand Russell, Écrits de logique philosophique, trad. Jean-Michel Roy, PUF, Paris 1989, p. 21.

4 René Lavendhomme, Lieu du sujet. Psychanalyse et mathématique, Seuil, Paris 2001, p. 8. Institut de philosophie, Centre de recherches scientifiques auprès de l'Académie slovène des sciences et des arts 
plicite de l'effacement devrait le nier comme effacement et devrait donc conduire à effacer l'effacement ${ }^{5}$.

« Ne serait-il pas possible, » demande le mathématicien René Lavendhomme, « qu'à l'intérieur de l'écriture même du texte mathématique se fasse jour la relation que ce texte entretient avec le sujet »? ${ }^{6}$ En proposant une autre définition de la mathématique selon laquelle « la mathématique est une science où l'on ne sait jamais de quoi l'on parle, ni si ce qu'on dit est vrai 7 ", Russell jette une lumière particulière précisément sur le lien que le mathématicien entretient avec la mathématique. Strictement parlant, les deux définitions russelliennes ne sont pas contradictoires. Si la première définition met l'accent sur le formalisme dont la rigueur logique bride la liberté dans le maniement des lettres et des symboles, la deuxième inclut une dimension supplémentaire, celle du dire mathématique qui met en relief le rapport des mathématiciens avec la mathématique. Le problème qui se pose alors est le suivant : qu'est-ce que la mathématique si le mathématicien peut ne pas y croire ? La légèreté de la lettre, corrélative au délestage du sens, libère-t-elle le sujet mathématicien à tel point qu'il se targue de ne pas savoir de quoi la mathématique parle, ni d'y croire vraiment?

Dans ce qui suit nous ferons référence aux commentaires respectifs de Badiou et de Lacan suscités par cet étrange énoncé affirmant que, en mathématique, on ne sait jamais de quoi on parle ni si ce que l'on dit est vrai. Badiou et Lacan insistent tous les deux sur un point, à savoir que le mathématicien n'est incontestablement pas libre de ne pas croire à la mathématique. Ou, pour reprendre les propres termes de Badiou, que « le mathématicien est d'abord celui qui croit 'dur comme fer' aux mathématiques ${ }^{8}$ ». Quant à l'ignorance mathématique, les réponses que donnent Badiou et Lacan divergent, et cela précisément dans la mesure où, même si l'accès à la psychanalyse, comme l'accès à la philosophie, est un certain type d'accès aux mathématiques, il ne s'agit tout de même pas du même type d'accès.

$5 \quad$ Ibid., p. 7.

6 Ibid.

7 Bertrand Russell, « Work on the principles of mathematics, » The International Monthly, 4, (1/1901), p. 84 .

8 Alain Badiou, «La mathématique est une pensée », Court traité d'ontologie transitoire, Seuil, Paris 1998, p. 43. 
Selon Badiou, lorsque Russell disait «que les mathématiques sont un discours où on ne sait pas de quoi on parle, ni si ce qu'on dit est vrai ", c'était « sans le croire, bien sûr ", et d'ailleurs " personne en vérité ne l'a jamais cru, sauf les ignorants, ce que n'était pas Russell ${ }^{»}$. Si Badiou ne peut pas croire Russell, c'est parce que, selon lui, les mathématiques sont, au contraire,

le seul discours qui «sache » absolument de quoi il parle : l'être, comme tel, quoique ce savoir n’ait nullement besoin d'être réfléchi de façon intra-mathématique, puisque l'être n'est pas un objet, ni n'en prodigue. Et c'est aussi le seul, c'est bien connu, où l'on ait la garantie intégrale, et le critère, de la vérité de ce qu'on dit, au point que cette vérité est l'unique jamais rencontrée à être intégralement transmissible ${ }^{10}$.

Cependant, Badiou semble être d'accord avec Russell sur un point tout à fait précis, à savoir : la mathématique ne sait pas et n'a pas besoin de savoir qu'elle fait de l'ontologie. Badiou affirme en effet que la mathématique en tant que discours sur l'être peut très bien se passer de ce savoir pour se perpétuer toute seule, presque à l'aveugle. Il revient donc à la philosophie de donner la « dignité ontologique ${ }^{11}$ » à ce que les mathématiciens se contentent de mettre au travail. Mais il y a plus important encore : cette ignorance est en quelque sorte constitutive de la mathématique en tant que science de l'être, puisque, comme le souligne Badiou,

il est de l'essence de l'ontologie de s'effectuer dans la forclusion réflexive de son identité. Pour celui-là même qui sait que c'est de l'être-en-tant-qu'être que procède la vérité des mathématiques, faire des mathématiques - et spécialement des mathématiques inventives - exige que ce savoir ne soit à aucun moment représenté. Car sa représentation, mettant l'être en position générale d'objet, corrompt aussitôt la nécessité, pour toute effectuation ontologique, d'être désobjectivante ${ }^{12}$.

\footnotetext{
9 Alain Badiou, L'Être et l'événement, Seuil, Paris 1988, p. 15.

10 Ibid.

$11 \quad$ Ibid., p. 21.

12 Ibid., p. 17.
} 
Après avoir posé que « ce qui est dicible - et dit - de l'être en tant qu'être ne relève d'aucune façon du discours philosophique ${ }^{13}$ » mais de la théorie des ensembles, la théorie cantorienne du multiple pur - après avoir reconnu, comme Russell, que les mathématiciens ne savent pas en tant que mathématiciens, pris dans l'exercice et l'épreuve de leur pratique, que ce qu'il font c'est de prononcer ce qui est dicible de l'être en tant qu'être - Badiou reconnaît également qu'il y a « les moments où la mathématique semble convoquée à se penser ellemême, à dire ce qu'elle est. » Il s'agit des moments singuliers où la mathématique - « sous la contrainte d'une butée réelle, ou du surgissement nécessaire, dans son champ, d'un point d'impossible » - semble requise, au regard de ses propres buts, de penser sa pensée. La mathématique n'est obligée de revenir sur elle-même que « sous l'injonction de sa butée intérieure ». Le moment de la torsion de la mathématique sur elle-même est le moment où la mathématique est confrontée « à sa dimension décisoire ${ }^{14}$ ».

Sur ce point, Badiou semble être obligé de distinguer deux modes opératoires de la mathématique identifiée à l'ontologie : il y a d'une part, le mode opératoire «normal » de la mathématique où la mathématique pense l'être sans pour autant se considérer comme « la pensée de la pensée qu'elle est ${ }^{15}$ », et, il y a, d'autre part, les moments de crise ou ${ }^{16}$ « la mathématique, butant sur un énoncé qui atteste en un point la venue de l'impossible, se retourne sur les décisions qui l'orientent ${ }^{17}$ ». C'est en s'appropriant un thème kantien, celui de l'orientation dans la pensée, que Badiou se propose de traiter la question de savoir si et dans quelles conditions la mathématique est capable de devenir la pensée de la pensée qu'elle est. L'orientation dans la pensée, dans le cas de la mathématique, porte sur la norme que se donne la pensée pour décider de l'existence. L'orientation dans la pensée porte, plus exactement, sur « ce que la pensée détermine en elle-même comme voie d'accès à ce qu'elle déclare exister ${ }^{18}$ ». Or, là encore,

13 Ibid., p. 20.

14 Badiou, «La mathématique est une pensée », pp. 48-49.

15 Badiou, «L'événement comme trans-être », Court traité de l'ontologie transitoire, p. 55.

16 Badiou évoque quelques exemples de ces moments critiques : crise des irrationnels dans la mathématique pythagoriciennne, la crise liée aux « paradoxes » de la théorie des ensembles, la crise liée aux théorèmes de limitation des formalismes, les polémiques suscitées par le statut de l'axiome du choix, etc. Voir Badiou, " La mathématique est une pensée », pp. 46-48.

${ }_{17} \quad$ Ibid., p. 51.

18 Ibid., pp. 49-50. 
le fait que la mathématique, dans les moments particuliers de crise, rencontre une impossibilité, un « bout de réel » ou se rapporte à sa propre pensée selon son orientation, est quelque chose dont la mathématique elle-même ne peut pas rendre compte, bien qu'elle le manifeste, comme le dit Badiou. Strictement parler, c'est à la philosophie sous condition des mathématiques, et plus particulièrement, à la philosophie, qui s'est mise sous condition de l'événementialité mathématique, puisqu'elle reconnaît la mathématique « comme un lieu de pensée singulier, dont les événements et procédures doivent être retracés dans l'acte philosophique ${ }^{19}$ » que revient la tâche de traiter la question : qu'est-ce qu'une orientation dans la pensée, lorsqu'il s'agit de la mathématique?

Pour cerner plus étroitement le partage des tâches entre la mathématique et la philosophie, il faut donc préciser que c'est parce que la mathématique - sauf dans les rares moments de «crise » - «pense l'être, mais n'est pas pensée de la pensée qu'elle est », qu'il revient à la philosophie "d'identifier la vocation


dans la mathématique que par la décision de la philosophie, c'est délimiter en même temps l'espace propre à la philosophie, celui de ce-qui-n'est-pas-l'êtreen-tant-qu'être, à savoir la vérité. Comment alors comprendre le lien entre l'ontologie et la philosophie, si « le dicible de l'être est disjoint du dicible de la véritée $^{21}$ » ? Si « toute vérité est postévénementielle ${ }^{22}$ », c'est-à-dire la production d'une nouveauté qui est en tant que telle insituable dans le système de savoirs connu, comme le soutient Badiou, et si « les mathématiques ne peuvent penser aucune procédure de vérité, puisqu'elles éliminent l'événement ${ }^{23}$ », ce multiple paradoxal qui échappe à l'ontologie, les mathématiques « doivent décider s'il est compatible avec l'ontologie qu'il y $a$ des vérités ${ }^{24}$ ». Et dans la mesure où l'être des vérités est conçu sous la forme de multiples non constructibles ou " génériques », la question que pose Badiou est la suivante : «l'ontologie peutelle produire le concept d'un multiple générique ? ${ }^{25}$ » C'est le mathématicien, Paul Cohen, qui a fourni la réponse à la question posée par la philosophie, dé-

\footnotetext{
19 Alain Badiou, Conditions, Seuil, Paris 1992, p. 158.

20 Badiou, «L'événement comme trans-être », p. 55.

${ }^{21}$ Badiou, L'Être et l'événement, p. 391.

22 Ibid.

23 Ibid., p. 376.

24 Ibid.

25 Ibid., p. 391.
} 
montrant l'existence des multiplicités " génériques », c'est-à-dire non constructibles. Ce que Badiou cherche et trouve dans la mathématique, c'est la preuve de « la compatibilité de l'ontologie avec la vérité ». Cette compatibilité implique, pour Badiou, que «l'être de la vérité, comme multiplicité générique, " peut et doit être « ontologiquement pensable, même si une vérité ne l'est pas ${ }^{26}$ ». C'est dans ce sens que nous nous proposons de lire la remarque à première vue assez obscure de Claude Imbert selon laquelle « Badiou pose que la mathématique cantorienne donne à la philosophie sa position de réel en même temps que les moyens de le penser ${ }^{27}$ ».

En revanche, pour Lacan, la nescience qui caractérise la mathématique selon Russell touche à ce que Lacan appelle « la frontière sensible entre vérité et savoir ${ }^{28}$ ". Dans son dialogue "improvisé » avec Russell, au cours de son séminaire « Le savoir du psychanalyste », Lacan clarifie ainsi la définition de Russell :

M. Bertrand Russell ... a pris soin de dire en ses propres termes [que] la mathématique, c'est très précisément ce qui s'occupe d'énoncés dont il est impossible de dire s'ils ont une vérité, ni même s'ils signifient quoi que ce soit. C'est bien une façon un peu poussée de dire que tout le soin précisément qu'il a prodigué à la rigueur de la mise en forme de la déduction mathématique, est quelque chose qui assurément s'adresse à toute autre chose que la vérité, mais a une face qui n'est tout de même pas sans rapport avec elle, sans ça il n'y aurait pas besoin de l'en séparer d'une façon si appuyée ${ }^{29}$.

C'est d'ailleurs dans ce contexte que Lacan introduit le problème de l'« incompréhension » des mathématiques. Cette incompréhension mathématique, pour utiliser son propre terme, permet à Lacan de poser la question suivante : quel ordre de vérité peut-on attendre des mathématiques ? Car l'incompréhension mathématique n'est pas une simple carence contingente mais doit être « considérée comme un symptôme » et Lacan en avance une raison :

26 Ibid.

27 Claude Imbert, « Où finit le platonisme? ", Alain Badiou : Penser le multiple, Charles Ramond (éd.), L'Harmattan, Paris 2002, p. 357.

28 Jacques Lacan, « Séminaire de Jacques Lacan, Le savoir du psychanalyste, 1971-1972 » (inédit), 4 novembre 1971.

29 Ibid., 2 décembre 1971. 
Les sujets en proie à l’incompréhension mathématique attendent plus de la vérité que la réduction à ces valeurs qu'on appelle, au moins dans les premiers pas de la mathématique, des valeurs déductives. Les articulations dites démonstratives leur paraissent manquer de quelque chose qui est précisément au niveau d'une exigence de vérité ${ }^{30}$.

Cette exigence de vérité est, selon Lacan, à la source de l'incompréhension mathématique, en tant qu'elle proviendrait d'un décalage, d'une insatisfaction devant une valeur de vérité réduite à la condition de la démonstration. Ceux qui ne comprennent pas les mathématiques auraient voulu qu'elles soient vraies, qu'elles disent quelque chose sur quelque chose, et, comme elles ne disent rien sur quoi que ce soit qui leur est extérieur, ${ }^{31}$ ils les rejettent en bloc. En d'autres termes, c'est parce qu'on veut du vrai que l'on ne comprend pas les mathématiques. Et, de façon plus ramassée : «Cette embrouille autour de l'incompréhension mathématique » signale le symptôme de «l'amour de la vérité ${ }^{32}$ ».

Ce qui paraît être l'évacuation de la pathétique du vrai laisse un vide, mais, à suivre Lacan : " on aurait tout à fait tort de penser que la mathématique est quelque chose qui, en effet, a réussi à vider tout ce qu'il en est du rapport à la vérité de son pathétique 33 ». Bien loin de réussir à éliminer sa « dimension du pathétique », l'histoire de la mathématique - à travers la crise des irrationnelles, l'apparition du calcul infinitésimal, les impasses des procédés de démonstrations, mais surtout « la peine, la douleur qu'a engendré, » ce que Lacan appelle «l'effraction cantorienne $» 34^{34}$ issue d'une rencontre avec la vérité qui a pu aller chez Cantor jusqu'à la menace de la folie - « de quelque rapports du mathème ...

30 Ibid.

${ }^{31}$ C'est parce que la formalisation mathématique « se fait au contraire du sens, j'allais presque dire à contre-sens. Le ça ne veut rien dire concernant les mathématiques, c'est ce que disent, de notre temps, les philosophes des mathématiques, fussent-ils mathématiciens eux-mêmes, comme Russell ». Jacques Lacan, Le séminaire de Jacques Lacan, Livre XX, Encore, Seuil, Paris 1975, p. 85.

32 Lacan, « Le savoir du psychanalyste », 2 décembre 1971.

33 Ibid.

34 Par exemple, lorsque Cantor établit une bijection entre l'ensemble des points d'un carré dont le côté est l'intervalle (o, 1), et cet intervalle lui-même, il écrit à Dedekind : « Je le vois, mais ne le crois pas ». C'est son système de croyance, plus précisément, son système d'interprétation, son ordonnancement symbolique, qui se trouve atteint. Cité dans Nathalie Charraud, Infini et Inconscient. Essai sur Georg Cantor, Anthropos-Economica, Paris 1994, p. 65. 
avec la dimension de vérité ${ }^{35}$ ». C'est exactement ce point qui intéressait Lacan, le rapport de la vérité et du savoir, où l'auteur d'une découverte, comme le montre bien le cas de Cantor, le « drame du savant ${ }^{36}$ ", se trouve lui-même nécessairement impliqué. La clarification de l'incompréhension mathématique importe pour la psychanalyse dans la mesure où la compréhension impliquerait sa réduction à des articulations non-contradictoires qui ne donnent pas sa place à ce que la vérité doit au désir. En effet, pour Lacan, « la non-contradiction ne saurait d'aucune façon suffire à fonder la vérité ${ }^{37}$ ». Or, c'est exactement sur cette question du lien entre le désir et la vérité que se produit le bouclage entre la mathématique et la psychanalyse. Mais il n'en est pas moins vrai que, si le désir est porteur d'une valeur de vérité qui excède le savoir mathématique, l'abord de celui-ci ramène au savoir mathématique ${ }^{38}$.

Si Lacan insiste sur la problématique de l'incompréhension, c'est aussi parce que l'incompréhension est gênante pour la psychanalyse dans la mesure où le travail de l'analyste, à la différence de celui du mathématicien, implique que, tout en sachant pratiquer la formalisation en analyse, l'analyste n'ait pas renoncé à la notion de vérité dont se passe quasiment le mathématicien. Ainsi, quand Lacan introduit le concept de mathème, c'est pour signaler « qu'il n'est absolument pas vrai de parler de mathème comme de quelque chose qui, d'aucune façon, serait détaché de l'exigence véridique ${ }^{39}$ ». Mais pour comprendre le caractère de vérité qui est à l'œuvre en analyse, pour comprendre le caractère de formalisation qui est au travail dans sa littéralité, il faut passer par les travaux qui tiennent la vérité pour une valeur parmi d'autres et qui s'effectuent fondamentalement en mathématiques.

\section{Deux modes du réel : le réel en mathématique, le réel en psychanalyse}

Ainsi, en mathématiques, « on commence par mettre des lettres sans dire absolument rien de ce à quoi elles peuvent servir $4^{\circ}$ », car pour penser il faut formali-

\footnotetext{
35 Ibid.

36 Jacques Lacan, « Science et vérité », Écrits, Seuil, Paris 1966, p. 870.

37 Ibid.

38 Sur ce point, nous renvoyons le lecteur au beau livre de Nathalie Charraud, Infini et Inconscient. Essai sur Georg Cantor.

39 Lacan, « Le savoir du psychanalyste », 2 décembre 1971.

$40 \quad$ Ibid., 15 décembre 1971.
} 
ser, c'est-à-dire donner une forme littérale à la pensée, travailler sur la littéralité de la pensée. Si d'un côté la notion même de mathématicité se trouve dépouillée jusqu'à une stricte littéralité pour mieux serrer le réel que vise la psychanalyse, c'est ainsi que nous pouvons décrire la manière selon laquelle Lacan aborde la mathématique, de l'autre côté, ce qui importe, selon Badiou, dans les projets mathématiques, à commencer par celui de Cantor, c'est d'instaurer « une formalisation intégrale, une théorie générale des univers de la pensée pure ... Réduire la mathématique à son acte : la puissance d'univocité du formalisme, la force nue de la lettre et de ses codes ». Ainsi, ce que vise la formalisation de l'acte mathématique, n'est rien d'autre que « le dire du réel mathématique ${ }^{41} »$.

C'est dans cette perspective que Badiou insiste sur la formalisation comme " une exigence de la pensée, aussi bien pour les mathématiciens que pour les philosophes », car ce qui importe à ses yeux, c'est que « la présentation formelle de la mathématique enveloppe une radicalité fondatrice quant à la nature de son acte ${ }^{42}$ ». Quelle leçon peut-on tirer de cette " passion pour la formalisation » qui caractérise les mathématiques du $20^{\text {ème }}$ siècle ? S’appuyant sur les démonstrations gödeliennes, Badiou met l'accent sur le fait que, même si « toute disposition formalisante de la pensée laisse un reste », la leçon à tirer de cet « échec » inévitable de la formalisation n'est pas qu'il faut abandonner la formalisation comme voie d'accès au réel, mais, au contraire, qu'il faut prendre le « résidu intraité » par une formalisation non aboutie comme point de départ pour une nouvelle formalisation :

C'est bien ce qui sépare la formalisation, comme pensée et projet, d'un simple usage pragmatique des formes. Il faut, sans jamais se décourager, inventer d'autres axiomes, d'autres logiques, d'autres manières de formaliser. L'essence de la pensée réside toujours dans la puissance des formes ${ }^{43}$.

Quant à Lacan, il est donc bien clair que ce n'est pas tant le savoir mathématique qui importe pour lui que la position du sujet-mathématicien par rapport à un désir inédit, celui de mettre un symbole, une lettre, là où il y a hors-sens. Finalement, ce qui l'intéresse dans les mathématiques, ce n'est pas la vérité,

\footnotetext{
${ }_{41}$ Alain Badiou, Le siècle, Seuil, Paris 2005, pp. 228-229.

42 Ibid., p. 230.

43 Ibid., pp. 230-231.
} 
mais leur puissance de construction, même au prix de voir la vérité réduite à une valeur parmi d'autres. C'est d'ailleurs en ce sens que Lacan a pu dire, ce qui à première vue peut paraître contradictoire, que : « Il n'y a de vérité que mathématique, c'est-à-dire écrite ${ }^{44}$ ». Car ce qui importe pour Lacan, tout comme pour Badiou d'ailleurs, dans les mathématiques, ce n'est pas seulement leur puissance structurante, celle de donner forme à ses objets, mais la puissance d'arrachement à l'expérience et à l'empiricité qui est attribuée aux mathématiques. ${ }^{45}$ Ainsi, selon Lacan, le discours mathématique ne peut se fonder sur rien d'autre que « ce langage de pur mathème ... le seul à pouvoir s'enseigner : ceci sans recours à quelque expérience, qui d'être toujours, quoi qu'elle en ait, fondée dans un discours, permet les locutions qui ne visent en dernier ressort rien d'autre qu'à, ce discours, l'établir ${ }^{46}$ ».

Pour Badiou, également, la mathématique s’intéresse « à la dimension la plus formelle, la plus abstraite, la plus universellement presque vide, de l'être comme tel ${ }^{47}$ ». Ce que la mathématique rend possible, en tant que « ressource spéculative », c'est ce que Badiou nomme « l'ontologie absolue », plus précisément, « l'existence d'un univers de référence, d'un lieu de la pensée de l'être en tant qu'être » qui ne se laisse « décrire, ou penser, qu'à partir d'axiomes, ou de principes, auxquels il correspond. Il n'en existe aucune expérience, ni aucune construction qui dépende d'une expérience. Il est radicalement non

44 Jacques Lacan, « Séminaire de Jacques Lacan XXII, R.S.I. 1972-1973», (inédit) 11 décembre 1972. Et plus précisément : «La mathématique fait référence à l'écrit, à l'écrit comme tel, et la pensée mathématique, c'est le fait qu'on peut se représenter un écrit. 》 « Séminaire de Jacques Lacan XXV, Le moment de conclure 1977-1978 » (inédit), 11 décembre 1978.

45 Ainsi, pour Platon, la mathématique est une condition du penser parce qu'elle rompt avec l'opinion. Or, dans la mesure où il s'agit d'une « rupture contrainte », comme le souligne Badiou, « involontaire, inapparente à elle-même, et surtout dépourvue de liberté », la mathématique, selon Platon, lu par Badiou, «n'établit pas la pensée dans la souveraine liberté de sa disposition propre ». Et plus précisément encore, définie comme «l'entre-deux de la vérité et de la liberté de la vérité », la mathématique est " la vérité encore captive de la non-liberté que réclame le geste violent de répudiation de l'immédiat. La mathématique appartient à la vérité, mais dans une figure contrainte de celle-ci ». Pour capter cette oscillation entre la vérité et la liberté, Badiou propose cette formule saisissante ; « la mathématique est trop violemment vraie pour être libre, ou elle est trop violemment libre (c'est-à-dire discontinue) pour être absolument vraie ». Alain Badiou, " Philosophie et mathématique », Conditions, 1992, pp. 168-171.

46 Jacques Lacan, «L'étourdit », Autres écrits, Seuil, Paris 2001, p. 472.

47 Alain Badiou, Éloge des mathématiques, Flammarion, Paris 2015, p. 43. 
empirique ${ }^{48}$ ». La mathématique crée donc des objets sans « s'appliquer » à l'expérience. Loin de poser l'existence comme extérieure à la mathématique, Lacan et Badiou la posent comme essentiellement déterminée par la mathématique elle-même. C'est dans ce sens que Lacan peut affirmer : «Il est clair que ce n'est qu'à partir d'une certaine réflexion sur les mathématiques que l'existence a pris son sens ${ }^{49}$ ». De même pour Badiou, la mathématique entendue en tant qu' " ontologie absolue » obéit au "principe de maximalité » selon lequel « toute entité intellectuelle dont l'existence s'infère sans contradiction des axiomes qui la prescrivent existe par cela même $\mathrm{e}^{50} »$.

Cette notion mathématique d'existence est lisible dans l'articulation de ce que Cantor nommait la formation correcte d'un concept. Dans « Les fondements d'une théorie générale des ensembles ", Cantor explique ce que l'on doit entendre par liberté de concevoir et de conceptualiser en mathématiques.

La mathématique est pleinement libre dans son développement et ne connaît qu'une seule obligation, ... ses concepts doivent être non contradictoires en euxmêmes et soutenir d'autre part avec les concepts déjà formés antérieurement, déjà présents et assurés, des relations fixes, réglées par les définitions. En particulier, pour pouvoir introduire de nouveaux nombres, elle est seulement requise d'en donner des définitions leur conférant une précision et le cas échéant une relation aux anciens nombres telles que l'on puisse dans des cas donnés les distinguer les uns des autres de manière déterminée. Dès qu'un nombre satisfait toutes ces conditions, il peut et doit être considéré comme existant et réel dans la mathématique ${ }^{51}$.

La liberté des mathématiques relève de la conception cantorienne de l'existence mathématique. La difficulté que Cantor confronte est celle de montrer que les nombres transfinis ont bien une existence effective, au même titre que les nombres finis. En liant existence et non contradiction, Cantor considère qu'il suffit qu'un objet mathématique ne contredise aucun énoncé de la théorie à la-

\footnotetext{
48 Alain Badiou, L'immanence des vérités, Fayard, Paris 2018, pp. 36-37.

49 Lacan, « Le savoir du psychanalyste », 1 juin 1972.

50 Badiou, L'immanence des vérités, p. 37.

${ }^{51}$ Georg Cantor, « Fondements d'une théorie générale des ensembles », paru en 1883 dans Mathematische Annalen, XXI ; pp. 545-586, trad. J.-C. Milner, Cahiers pour l'analyse, no. 10, p. 48.
} 
quelle il appartient pour exister, il n'est donc pas besoin d'une démonstration effective de son existence.

Conscient de rompre avec les mathématiques traditionnelles, Cantor insiste sur le fait qu'il ne peut « poursuivre [ses recherches sur les nombres transfinis] qu'en étendant au-delà de ses limites antérieures le concept de nombre entier existant réellement. En vérité, cette extension s'oriente dans une direction où, à ma connaissance, nul ne l'avait jusqu'à présent cherchée ${ }^{52} »$. Or cette nouvelle exigence de considérer les nouveaux nombres, les transfinis, comme existant réellement, se fonde sur la notion même de nombre : « La notion de nombre, si développée qu'elle soit ici, porte en soi le principe d'une extension nécessaire en elle-même et absolument infinie ${ }^{53}$ ». Ainsi, pour Cantor, les nombres transfinis sont aussi « réels » que les entiers finis ou les irrationnels. Car le transfini n'est pas simplement un infiniment grand, un «fini variable ", « une grandeur pouvant croître au-delà de toute limite finie ${ }^{54} »$. Si le transfini est un infini actuel ou infini proprement dit, c'est parce qu'on peut

le fixer de façon mathématique par des nombres, cette pensée s'est imposée à moi logiquement, presque contre ma volonté ... L'assomption qu'en dehors de l'absolu, de ce qui ne peut être atteint par aucune détermination, et du fini, il ne devrait pas exister de modifications qui soient déterminables par des nombres, encore que non-finies, et soient par conséquent ce que j'appelle l'infini proprement dit - cette assomption ne me paraît justifiée par rien ... Ce que j’affirme et crois avoir démontré par le présent travail ainsi que par mes tentatives antérieures, c'est qu'après le fini, il existe un transfinitum (que l'on pourrait aussi nommer suprafinitum), c'est-à-dire une échelle illimitée de modes déterminés qui par nature ne sont pas finis, mais infinis, et qui cependant peuvent être précisés, tout comme le fini, par des nombres déterminés, bien définis et distinguables. Ma conviction est dès lors que le domaine des grandeurs définissables n'est pas clos avec des grandeurs finies et que les limites de notre connaissance peuvent être étendues en conséquence, sans qu'il soit nécessaire pour autant de faire violence à notre nature ${ }^{55}$.

$52 \quad$ Ibid., p. 35.

53 Georg Cantor, Gesammelte Abhandlungen mathematischen und philosophischen Inhalts, E. Zermelo (éd.), Springer, Berlin 1932, p. 95.

54 Ibid., p. 374.

55 Cantor, «Fondements d'une théorie générale des ensembles », pp. 42-43. 
Ainsi se trouve réfutée une thèse traditionnellement opposée à l'infini actuel en mathématiques : la finitude de notre entendement nous empêche de concevoir l'infini qui se situerait entre le fini et l'absolu (Dieu). La thèse défendue par Cantor est que

si bornée que soient en fait la nature humaine, il y a cependant en elle une très grande part d'infini, et je vais jusqu'à soutenir que si elle n'était pas elle-même infinie sous bien des rapports, on ne saurait expliquer la conviction et la certitude assurées où nous nous savons tous unis, touchant l'être de l'absolu. En particulier, je tiens que l'entendement humain est doué d'une aptitude illimitée à former par progression des classes de nombres entiers, qui soutiennent une relation déterminée avec les modes infinis et en constituent les puissances de degré croissant ${ }^{56}$.

Comme tous les mathématiciens ou presque, Cantor adopte une forme de réalisme du nombre. Il fait la distinction entre la réalité intrasubjective et la réalité transsubjective : La première réalité, dite « intrasubjective ou immanente » relève de notre entendement. Mais il y a une autre réalité des nombres que Cantor appelle « transsubjective ou transcendante » ce qui implique que les nombres ont une réalité hors de l'entendement, puisqu'ils sont « comme expression ou reproduction de processus et de relations existant dans le monde extérieur opposé à l'intellect ${ }^{57}$ ». Dans la mathématique, telle que la conçoit Cantor, la réalité immanente prime sur la réalité transsubjective ou transcendante, cette dernière étant assignée à la métaphysique. Or, le fait que, dans la mathématique, il faut " prendre en considération... uniquement et seulement la réalité immanente », permet d'en tirer " une conséquence importante pour la mathématique ${ }^{58}$ »: le fait qu'elle ne doit s'occuper que de la réalité immanente de ses objets, lui accorde une liberté que les autres sciences ne connaissent pas. Mettant ainsi au centre de sa conception des mathématiques sa liberté de création, Cantor adopte en même temps l'idée que la mathématique pense à travers la formalisation, mais cela suppose qu'il y a un système d'axiomes qui ne porte pas sur des objets qui existent dans la réalité, mais sur des objets que les axiomes-prescriptions appellent à l'existence.

\footnotetext{
56 Ibid., p. 44 .

57 Ibid., p. 47.

${ }_{58}$ Ibid., p. 48.
} 
Badiou, pour sa part, souscrit au réalisme des mathématiciens. En effet, si les mathématiques sont la science du réel, c'est parce que la philosophie, qui se fonde sur l'équation la mathématique = l'ontologie, part de " la supposition qu'il y a dans ce qui existe un niveau de généralité ou d'universalité qui est en quelque sorte immatériel. Il y a des structures qui se retrouvent dans tout ce qui existe. L'étude de ces structures en tant que telles, des possibilités structurales, est précisément l'enjeu des mathématiques ${ }^{59}$ ». Tout comme les mathématiciens, Badiou croit que les objets et structures mathématiques « 'existent' en un certain sens ». Mais, à la différence des mathématiciens, le réalisme de Badiou se base sur l'expérience d'une résistance dans le travail mathématique, à savoir « que 'quelque chose' résiste lorsqu'on fait des mathématiques, qu'on se frotte à une réalité difficile, rebelle ${ }^{60}$ ». C'est à partir de la résistance des « choses » à la pensée qu'il faut saisir le réel en mathématiques. Tout le problème est d'identifier ce « bout de réel » qui se manifeste seulement là où ça coince, là où il y a une impasse, une incompatibilité.

Badiou rejoint ainsi Lacan, pour qui, ce n'est pas seulement par les mathématiques que l'on pense le mieux l'existence. L'existence est ce que les mathématiques pensent dans sa pointe, c'est-à-dire dans son impossibilité. Si la notion d'impossible joue le rôle d'un pivot essentiel qui organise les rapports que la psychanalyse entretient avec les mathématiques, c'est parce que, comme le discours psychanalytique, le discours mathématique rencontre des points d'impossible. Située à l'intersection de la psychanalyse et des mathématiques, la notion d'impossible permet d'ordonner le champ psychanalytique d'une part, et de révéler, d'autre part, les impasses constitutives des mathématiques. L'impossible n'est donc pas tout à fait étranger aux mathématiques puisqu'il est incarné dans leur histoire par de grands moments de « crise » où elles se heurtent contre un réel énigmatique qui correspond à la rencontre d'un obstacle excédant le cadre axiomatico-déductif.

Mais il faut noter cependant que le réalisme lacanien est bien singulier puisqu'il dépend de sa définition du réel comme impossible. Le réel n'est pas la réalité, mais ce qui se soustrait à sa représentation. Il est ce qui fait trou dans un discours et le pousse à se déplacer et se renouveler. Et c'est précisément pour

59 Badiou, Éloge des mathématiques, p. 59.

60 Ibid., pp. 72-73. 
mieux cerner l'impossible dans la psychanalyse même que Lacan fait appel aux mathématiques. Ce que Lacan cherche dans les mathématiques, c'est une écriture, car le réel en jeu dans la psychanalyse ne s'atteint que par les impasses des voies de l'écriture. En choisissant d'emprunter aux mathématiques les outils pour le travail à faire en tant que psychanalyste, convaincu « de la valeur des éléments mathématiques pour faire émerger quelque chose qui concerne vraiment l'expérience de l'analyse ${ }^{61} »$, ce n'est pas seulement pour rompre avec le discours philosophique que Lacan a recours aux mathématiques. C'est parce que les mathématiques ont affaire au réel, qu'elles sont les seules à penser le réel : «Le Réel dont je parle est absolument inapprochable, sauf par une voie mathématique » et « c'est très précisément en cela que l'effort logicien doit nous être un modèle, voire un guide ${ }^{62} »$.

Comme les mathématiques, la psychanalyse ne trouve pas sa limite à l'extérieur d'elle-même, mais la porte en soi. ${ }^{63}$ Aborder le réel sous l'angle de la limite nous donne, une fois de plus, l'occasion de préciser un point fondamental des rapports de la psychanalyse avec les mathématiques. Nous nous proposons d'illustrer ce paradoxe de la limite à partir de la notion d'infini. Si Lacan a recours aux mathématiques et s'il fait de Cantor un interlocuteur privilégié, c'est aussi pour résoudre l'impasse de Freud, celle qui touche à la fin de l'analyse. En tant qu'expérience de parole, la psychanalyse, de structure, ne connaît aucune fin. Or si, pour Freud, l'analyse est « interminable », sans fin, puisqu'il n'y a rien qui puisse arrêter le déchiffrage de l'inconscient, Lacan, pour sa part, propose une notion de la fin « logique » de l'analyse. C'est dans cette perspective que la fin de l'analyse, selon Lacan, met la psychanalyse face à un enjeu majeur : «L'enjeu maintenant est de quoi aidera à sortir le réel-de-la-structure : de ce qui de la langue ne fait pas chiffre, mais signe à déchiffrer ${ }^{64}$ ». Faute d'être muni des outils mathématiques, seuls à même de localiser le point d'impossible, l'analyse ne rencontrera aucun réel.

\footnotetext{
${ }_{61}$ Lacan, « Le savoir du psychanalyste », 1 juin 1972.

62 Ibid., 2 décembre 1971.

${ }_{3}$ "Si l'inscription analytique est bien ce que j'en dis, à savoir le début, le noyau-clé de sa mathématique, il y a toutes les chances à ce que ça serve à la même chose que la mathématique. C'est-à-dire que ça porte en soi sa propre limite. » Lacan, « Séminaire XXII », 20 novembre 1973.

64 Jacques Lacan, « Télévision », Autres écrits, p. 536.
} 
La découverte cantorienne des transfinis va lui permettre d'élaborer une théorie de la fin de l'analyse en y incluant son infinitude. Et c'est d'ailleurs dans ce but qu'il a proposé la fameuse procédure de la " passe » qui marque le passage de l'analysant à l'analyste. La psychanalyse qui vise sa fin a pour ambition de cerner, à la fin de l'analyse, ce qui détermine le sujet à son insu. La rigueur de cette détermination dépend d'une structure qui participe du réel. Ce point de réel qui ne se manifeste qu'à la fin de l'analyse, se fonde sur la construction d'un cadre qui est censé englober tous les dits de l'inconscient du sujet. On voit bien l'analogie du franchissement qu'implique la passe lacanienne et le geste de Cantor. Or dans la psychanalyse, «il est question de l'accès à une formule, un $\aleph_{0}$, qui est celui de chacun, même s'il y a un $\aleph_{0}$, pour tous que Lacan a formulé dans les termes 'Il n'y a pas de rapport sexuel' ${ }^{65}$ ».

Le fait de pouvoir toujours ajouter des dits nouveaux, signale que l'on est dans un infini que Cantor qualifie d' "improprement dit ». Dans ce contexte, la cardinalité du symbolique, le terrain propre à la psychanalyse, sera toujours finie. Or l'infini proprement cantorien, l'infini actuel, n'est atteint qu'à partir de la rupture avec une notion de l'infini réduit à l'inaccessible, l'indéfini ou l'illimité, rupture qui a permis à Cantor de construire un infini purement quantitatif : les nombres transfinis. Pour soutenir cette opposition inhérente à la psychanalyse, il faut donc se tourner vers la théorie mathématique de l'infini. On commence par admettre que, dans la psychanalyse, le symbolique est infini, puisqu'il est toujours possible d'ajouter un dit supplémentaire à tous les dits précédents. Si Lacan fait appel à Cantor, c'est pour s'appuyer sur sa découverte des nombres transfinis, car la théorie des transfinis lui permet de penser la façon de dépasser le Nom-du-Père, le rôle pivot de la castration finitiste, qui pour Freud présente un point indépassable pour la psychanalyse, de relativiser cette place qui jusqu'alors avait un caractère absolu.

La théorie des nombres transfinis intéresse Lacan dans la mesure où elle propose une progression de nombres infinis qui ne rencontre aucune butée. De même que le problème pour Cantor est celui de ne pas pouvoir sortir du dénombrable pour atteindre la puissance du continu, le déchiffrage de l'inconscient ne peut atteindre sa fin. Le pas de Cantor, pour faire le saut du dénombrable au continu, consiste à comprendre qu'on ne peut dépasser qu'en limitant au

65 Jacques-Alain Miller, « Vers un signifiant nouveau », Revue de l'ECF, (20/1992), p. 54. 
préalable. C'est par l'adjonction d'un nouveau signifiant, aleph zéro, un nombre ajouté à la suite infinie de nombres et le nommant, que le fini se saisit. La mathématique cantorienne présente l'intérêt d'avoir formalisé non seulement la notion de limite, mais encore celle des dépassements successifs de limitations successives. Pour atteindre des cardinaux toujours plus grands, il faut appliquer ce que Cantor appelle « le principe de limitation ». Une fois le premier passage à la limite accompli, il faut donc un nombre transfini de limitations pour envisager l'ultime saut, impossible à écrire parce qu'il est toujours possible d'en rajouter « un en plus » et donc de le dépasser. Le paradoxe du "plus grand transfini » rend la place de tous les ensembles inconsistante. Or, c'est précisément l'une des possibles fins de l'analyse, à savoir la rencontre avec le manque du dernier signifiant au cœur du symbolique, l'incomplétude de l'Autre symbolique mais qui ouvre la possibilité de « l'invention d'un savoir dans le réel » que Lacan propose comme " une détermination du réel au même titre que la science $^{66} »$. L'enjeu pour la psychanalyse est de (se) rendre compte qu'en choisissant un modèle d'infini on décide ce qu'il faut entendre par la notion d'infini. Il faut toutefois noter que c'est seulement le premier modèle de l'infini, celui qui se limite au dénombrable, qui est utilisable en psychanalyse. À cet égard, si Badiou reproche à Lacan de ne pas être cantorien ${ }^{67}$, car l'infini pour lui se réduit à l'inaccessibilitée ${ }^{68}$, Lacan ne pourrait que lui donner raison dans la mesure où, tout comme Freud, il n'admet pas l'infini actuel dans la psychanalyse ; ils sont tous deux foncièrement « finitistes ».

Ainsi, l'impossible d'écrire est propre aux mathématique tout autant qu'à la psychanalyse. On comprend mieux pourquoi le mathème est « le point pivot » de l'entreprise lacanienne. C'est que la voie du mathème, sous la forme même où Lacan la propose, est la seule méthode possible à produire des « petits bouts de réel ", comme il le dit lui-même. Le paradoxe est que l'on ne peut produire des « bouts de réel » que par des artifices, très exactement, par ceux de l'écriture. Au paradoxe de voir le réel dans l'écriture, se joint celui de ne voir appa-

\footnotetext{
66 Jacques Lacan, « La note italienne », Autres écrits, p. 310.

${ }_{67}$ Selon Badiou, la doctrine lacanienne du sujet est essentiellement finie et il en avance la raison: « Jusque dans la logique de la jouissance, la réelle existence de l'infini actuel l'encombre plus qu'elle ne le sert. Il ne convoque l'infini que pour le révoquer. L'infini doit rester cette fiction opératoire, qui pointe l'abîme ou la faille où le sujet se constitue. » Badiou, «Sujet et infini », Conditions, p. 301.

68 Ibid., p. 300.
} 
raître le réel que par l'écriture. Et pour donner une image qui se rapproche le plus de cette formalisation qui ne se supporte que de l'écrit, de cette « réduction aux dimensions de la surface qu'exige l'écrit », Lacan prend une image qu'il trouve dans la nature,

ce travail de texte qui sort du ventre de l'araignée, sa toile. Fonction vraiment miraculeuse, à voir, de la surface même surgissant d'un point opaque de cet étrange être, se dessiner la trace de ces écrits, où saisir les limites, les points d'impasse, de sans-issue, qui montrent le réel accédant au symbolique ${ }^{69}$.

Sur ce point la philosophie ne semble pas être en désaccord avec la psychanalyse. Pour Badiou, les mathématiques « seraient un mode d'approche du réel, y compris le plus insaisissable ${ }^{70}$ ». Mais le réel que viseraient les mathématiques selon Badiou n'est pas tout à fait le même que celui que visent les mathématiques selon Lacan. Pour ce dernier, "Le réel ne saurait s'inscrire que d'une impasse de la formalisation ${ }^{71}$ ». Plus précisément, c'est seulement à mettre la formalisation à l'épreuve qu'on rencontre un point d'impossibilité. Cependant il faut souligner également que le formalisme de Lacan est très particulier par bien des aspects. Le fait de prendre la formalisation mathématique comme modèle ne revient pas pour autant à confondre les deux modes du réel : le réel qui est en jeu dans la mathématique et le réel que vise la psychanalyse. La mathématique, et la science en général, ont affaire avec un réel qu'elles transforment en écriture. Le réel dont il s'agit ici est censé être mathématisable - sans reste. La psychanalyse, en revanche, étant affaire de sens, ne donne pas du sens au réel. Il s'agit plutôt de viser le réel dans le sens. La mathématique représente donc pour Lacan le modèle d'accès au réel de la structure où le réel est saisi au sens de rencontre d'un point d'impossible à écrire dans les termes de cette structure. Dans le séminaire L'envers de la psychanalyse, Lacan examine une formulation de l'impossible comme fait de structure, justement : « À poser la formalisation du discours et, à l'intérieur de cette formalisation, à s'accorder à soi-même quelques règles destinées à la mettre à l'épreuve, se rencontre un

\footnotetext{
Lacan, Encore, pp. 85-86.

70 Badiou, Éloge des mathématiques, p. 59.

${ }^{71}$ Lacan, Encore, p. 85.
} 
tel élément d'impossibilité ${ }^{72}$ ». L'épreuve est prise ici au sens où elle atteint un certain réel.

\section{Le dire de Cantor selon Lacan et Badiou : « un pari sur le réel 73 ॥}

C'est dans ce contexte que Badiou critique certains aspects de l'usage des mathématiques par Lacan. Nous ne saurions entrer ici dans le détail d'une discussion de ses arguments tout à fait recevables de Badiou, qui s'appuient sur une lecture attentive de « L'étourdit » et de Encore. Avant même de s'interroger sur le bien-fondé de sa lecture et/ou critique de Lacan, surtout sur ces points où Lacan s'appuie sur les mathématiques, il faut se demander à quoi la lecture de Lacan peut servir dans le projet philosophique construit par Badiou. Certes, il y a chez Lacan la voie du mathème pour situer la question du réel qui semble précieuse à Badiou puisque lui aussi vise à cerner « ce qui se soustrait à la détermination ontologique », « ce qui n'est pas l'être-en-tant-qu'être ${ }^{74}$ » et qui n'est donc pas mathématisable. Car le problème que Badiou se propose de résoudre est le suivant : « si l'ontologie réelle se dispose comme mathématique en éludant la norme de l'un, il faut aussi, sauf à rétablir globalement cette norme, qu'il y ait un point où le champ ontologique, donc mathématique, se détotalise, ou reste en impasse. Ce point, je l'ai nommé l'événement $t^{75}{ }^{\prime}$. Ainsi, si la philosophie se sépare d'une partie de soi-même, celle qui s'interroge sur l'être comme tel, en assignant cette tâche à la mathématique, c'est aussi pour s'établir comme " théorie générale de l'événement. C'est-à-dire de ce qui se soustrait à la soustraction ontologique. Ou théorie de l'impossible propre des mathématiques ${ }^{76}$ ». Mais pour assigner à la philosophie la tâche de déterminer le trans-être de l'événement, il faut commencer par admettre que « tout n'est pas mathématisable ${ }^{77}$ ». La question qui se pose alors est celle de savoir qu'est-ce qui dans l'événement mérite le nom du réel proprement dit, le réel en tant qu'impossible d'écrire.

C'est précisément sur ce point que Badiou ne peut pas ne pas rencontrer Lacan dans sa tentative d'aborder le réel ininscriptible à partir de la mathématique.

\footnotetext{
72 Jacques Lacan, L'envers de la psychanalyse, Seuil, Paris 1991, p. 50.

73 Alain Badiou, Théorie du sujet, Seuil, Paris 1982, p. 290.

74 Badiou, «L'événement comme trans-être », p. 56.

75 Ibid., pp. 56-57.

${ }^{76}$ Ibid., p. 57.

77 Ibid.
} 
C'est aussi l'occasion de poser la question suivante : pourquoi faut-il avoir recours au mathème en psychanalyse ? Si la psychanalyse, comme tout discours visant un réel, s'articule en mathèmes, c'est parce qu'elle butte sur une difficulté : «Sa difficulté tient à ce que, comme savoir, elle traite d'un savoir, lui aussi littéralisable, mais qui ne se sait pas $^{78}$ ». La psychanalyse n'est pas la mathématique bien qu'elle privilégie, tout comme la mathématique, la voie du mathème pour accéder au réel, mais, à la différence de la mathématique, elle vise le résidu de réel qui échappe à toute formalisation. Pour la psychanalyse ce résidu de réel qui n'est pas mathématisable se résume en la formule qu' «il n’y a pas de rapport sexuel » : il n'y a qu'impossibilité à écrire le 2 d'un rapport entre deux ensembles sexués. Or c'est précisément ce point d'impossible qui soutient un désir de savoir. Il ne s'agit pas d'un désir de savoir malgré tout, malgré cet impossible, mais d'un désir reposant sur le fait que la vérité n'est pas toute, qu'elle peut s'agrandir, faisant place à de nouveaux savoirs ${ }^{79}$. Lacan propose de tenter l'écriture du rapport sexuel, bien qu'il en ait avancé l'impossibilité :

Sans essayer ce rapport de l'écriture, pas moyen en effet d'arriver à ce que j'ai, du même coup que je posais son inex-sistence, proposé comme un but par où la psychanalyse s'égalerait à la science : à savoir démontrer que ce rapport est impossible à écrire, soit que c'est en cela qu'il n'est pas affirmable mais aussi bien non réfutable : au titre de la vérité. Avec pour conséquence qu'il n’y a pas de vérité que l'on puisse dire toute... . La vérité ne sert à rien qu'à faire la place où se dénonce ce savoir. Mais ce savoir n'est pas rien. Car ce dont il s'agit, c'est qu'accédant au réel, il le détermine tout aussi bien que le savoir de la science ${ }^{80}$.

Ce qui est visé dans les mathèmes lacaniens, ce n'est donc ni de l'ordre du vrai ni de l'ordre du savoir, car le mathème est situé à la jonction de la vérité et du savoir, mais le réel qui se manifeste sous forme de la rencontre, du coinçage, de l'incompatible, du non-rapport. C'est pourquoi ce que vise le mathème, ce n'est pas le réel en tant que tel mais l'impossibilité de dire vrai du réel. C'est là que la manière dont Lacan utilise les mathématiques est à prendre au sérieux.

\footnotetext{
Lacan, Encore, p. 88.

79 Lacan, « Note italienne, » p. 310.

80 Ibid.
} 
La voie du mathème, pour situer la question du réel, rejoint le cœur des préoccupations de Badiou et, plus particulièrement, dans sa tentative pour réintriquer la philosophie et les mathématiques. Il nous faut nous interroger sur le point exact où les chemins de Lacan et Badiou se séparent tout en se croisant parfois. Dans la perspective de Lacan, le fait de s'inspirer du modèle mathématique, ne revient pas pour autant à confondre deux modes du réel : un réel qui fait travailler les mathématiciens, le réel en tant que cause du travail de formalisation, et un réel qui est produit par cette formalisation ou, plus exactement, par son échec. Il faut d'abord souligner que le réel est toujours relatif à un discours, car on ne tombe sur le réel qu'à partir d'un discours. Il en découle que l'émergence d'un nouveau réel a pour conséquence le remaniement du discours mathématique. Cela paraît contredire la philosophie spontanée des mathématiciens, le " réalisme platonicien » qui présuppose un univers mathématique préétabli que les mathématiciens n'ont plus qu'à découvrir. Or, si le réalisme des mathématiciens et la production de nouveaux réels ne sont pas incompatibles, c'est parce qu'une fois produit, le nouveau réel est posé comme étant déjà là.

C'est la leçon qu'on peut tirer de l'invention des transfinis : une fois les nouveaux nombres, les transfinis, construits par Cantor et introduits dans le champ mathématique, tout se passe comme s'il n'avait fait que les découvrir, puisque ils étaient toujours déjà là. Ainsi, ce qui n'est que produit d'une invention, est considéré, après coup, comme étant déjà là avant les démarches de Cantor qui l'ont engendré. Cette inversion de l'invention en découverte est le prix à payer pour le réalisme des mathématiciens. Celui-ci implique cette sorte de rétroaction pour garantir la « réalité » de ses objets. C’est que la réalité mathématique s'impose à tous comme étant nécessaire. Pour traiter du réel mathématique, il faut donc prendre comme point de départ le fait qu'un réel se produit comme hors de toute juridiction. Ainsi, pour produire les transfinis, il fallait inventer le chemin pour atteindre l'infini mathématique. La méthode de diagonale de Cantor était contingente, donc non requise par le discours mathématique existant. Il fallait donc l'inventer, trouver le chemin qui n'était pas donné. La méthode n'était pas nécessitée par le savoir mathématique au moment de son apparition, et pour cause, puisque l'invention du chemin, de la méthode, visait à résoudre une faille dans ce savoir même. Cependant, une fois l'invention des transfinis admise par le discours mathématique, cette contingence devient nécessaire. 
On serait donc plutôt d'accord avec Badiou, lorsqu'il avance que le dire mathématique, en tant qu'événement, ne se soutient d'aucune garantie de la nécessité $^{81}$. Pour comprendre cette articulation paradoxale, déjà évoquée par Cantor, entre la liberté de création et la contrainte ${ }^{82}$ qui caractérise la mathématique, il faut préciser que, bien qu'il soit produit par le discours mathématique, le réel des mathématiques, pour être effectif, c'est-à-dire capable de renouveler le savoir mathématique, doit être posé comme ex-sistant au discours mathématique, et même comme étant préalable, hors de ce discours dont il procède cependant. Sur cette question de l'ex-sistence il faut spécifier que n'ex-siste que ce qui se manifeste comme une impossibilité à prendre ici au sens qu'il est impossible que ce réel puisse être affirmé ou nié par la démonstration mathématique. Ce qui revient à dire que le statut de réel de quelque chose qui se présente comme une entrave ou une difficulté dépend de la démonstration mathématique. On pourrait dire aussi que le réel comme ex-sistence, c'est une vérité démontrée comme impossible à démontrer. C'est précisément à ce qu'il ne puisse s'écrire que l'on reconnaît ce réel en mathématiques. L'exemple notoire qui, pour Lacan, illustre bien le réel en mathématiques, c'est l'impossibilité de démontrer la validité de l'hypothèse du continu. Au lieu de la démontrer par la méthode diagonale, Cantor a fait émerger l'hypothèsee du continu comme le réel-impossible des mathématiques. Plus généralement donc, si toute démonstration, une fois validée, implique qu'il est impossible de procéder autrement, l'impossibilité de la démonstration, c'est-à-dire l'impasse de la formalisation, l'impossibilité à écrire ou à ne pas écrire une formule, en revanche, génère un réel en tant qu'impossible.

C’est à ce niveau là que se situe la critique que Badiou adresse à Lacan. Pour aborder le rapport singulier de Lacan aux mathématiques, Badiou prend comme point de départ ce qu'il appelle « la triangulation » qui inclut la psychanalyse, la mathématique et la philosophie ${ }^{83}$. Badiou s'appuie sur un repère tiré de « L'étourdit » où Lacan met l'accent sur la défaillance de la philosophie lorsqu'il s'agit d'identifier l'essentiel de la mathématique : « Pour être le langage le plus propice au discours scientifique, la mathématique est la science sans

\footnotetext{
81 Alain Badiou, Le Séminaire. Lacan. L'antiphilosophie 3, 1994-1995, Fayard, Paris 2013, p. 126.

82 Ibid., p. 129.

$83 \quad$ Ibid., p. 39.
} 
conscience dont fait promesse notre bon Rabelais, celle à laquelle un philosophe ne peut que rester bouché ${ }^{84} »$. En revanche, ce qui caractérise l'approche de la psychanalyse à la mathématique tient au statut singulier que Lacan attribue au mathème : d'être vidé du sens, le mathème assure la transmission intégrale d'un savoir. Ainsi, pour Lacan, le discours mathématique, défini comme « langage de pur mathème » est " ce qui est seul à pouvoir s'enseigner : ceci sans recours à quelque expérience ${ }^{85}$ ». C'est ce côté que le mathème en psychanalyse et le mathématisable aurait en commun. Mais il y a aussi un autre côté du mathème qui va au-delà du mathématisable. Pour la psychanalyse, c'est le réel du « il n'y a pas de rapport sexuel », le réel non-inscriptible, mais autour duquel tout dans le discours psychanalytique s'ordonne. C'est aussi ce réel qui ne peut être signalé que par les impasses du mathématisable : «C'est en quoi les mathèmes dont se formule en impasse le mathématisable, lui-même à définir comme ce qui du réel s'enseigne, sont de nature à coordonner à cette absence prise au réel ${ }^{86}$ ». "L'absence prise au réel » évoquée ici, n'est rien d'autre que le réel propre à la psychanalyse, le « il n'y a pas de rapport sexuel », qu'il faut distinguer du réel inscriptible et donc enseignable par la voie du mathème. C'est ce réel dont l'absence est, comme le dit Badiou, « absence dans aucune mathématisation, c'est-à-dire son absence dans l'inscription ${ }^{87}$ ».

Quel serait l'équivalent de cette « absence dans l'inscription », pour les mathématiques ? La " réalité » des mathématiques tient au fait que leur cohérence s'impose comme contrainte. Et si l'on peut dire que la formalisation revient à une mise en rapport, le réel survient comme impossibilité de mise en rapport, comme rapport impossible. Par conséquent, le réel en mathématique ne peut émerger que dans les lieux où cette cohérence est mise en défaut. C'est à ce niveau-là que Lacan cherche le réel en mathématique : le réel dans la formalisation même. Le réel dont il s'agit ici est celui qui « ne saurait s'inscrire que d'une impasse de la formalisation ». Ce qui est important à noter ici, c'est que c'est précisément sur ce point que Badiou avance ses objections à Lacan. La position de Lacan serait, selon Badiou, une position « $\operatorname{archiscientifique}^{88}$ ». À suivre la lecture badiousienne de l'articulation que Lacan propose du réel et du

84 Lacan, «L'étourdit », p. 453.

85 Ibid., p. 472.

86 Ibid., p. 479.

87 Badiou, Lacan, p. 43.

88 Ibid., p. 45. 
mathème, celle-ci n'est tenable que si l'on n'introduit pas le point d' " un réel du réel ». La formule : le « réel du réel ${ }^{89}$ » qu'on ne trouvera nulle part chez Lacan, comme Badiou l'admet volontiers, tient une place stratégique dans son argumentation à lui, puisqu'elle lui permet de clarifier comment se positionnent respectivement, selon Lacan, la psychanalyse et la philosophie par rapport à la mathématique.

Ainsi, à suivre Badiou, pour situer la place de la psychanalyse par rapport à la mathématique et à la science en général, il lui faut redoubler le réel, le réel propre à la science, le réel dont la science« découvre » les lois et qu'elle inscrit en formules, le réel mathématisable et transmissible par la voie du mathème enseignable ; et le réel propre à la psychanalyse, le réel non-mathématisable qui ne peut s'inscrire que par les impasses de la mathématisation. Ce que cherche Lacan, d'après Badiou, c'est précisément ce point d'impossible dans la mathématique elle-même qui permet de cerner le réel non mathématisable. Le mathème, tel que le conçoit Lacan, est archiscientifique et non pas scientifique, selon Badiou, parce qu'il est situé au point du réel de la mathématique. Et Badiou de conclure, «il ne peut pas être mathématique, précisément parce qu'il touche au réel de la mathématique elle-même ${ }^{90} »$. Or, c'est précisément cette distinction entre deux modes du réel qui séparent la psychanalyse de la philosophie qui, n'étant pas capable de toucher au réel de la mathématique, reste, comme l'affirme Lacan, « bouchée aux mathématiques ». Le fait d'avoir accordé le statut de pensée à la mathématique, seule à toucher le réel, est en même temps l'occasion pour Lacan de critiquer la philosophie qui, elle, est incapable de reconnaître, dans la mathématique, la voie d'accès au réel qui s’y trouve, et ne peut pour cette raison même que d'être bouchée à la mathématique.

Si Badiou rejette en bloc cette idée, c'est parce qu'il défend la thèse selon laquelle « s'il y a un lieu de pensée qui est bouché à lui-même, c'est bien la mathématique » et la raison qu'il avance n'est rien d'autre que son ignorance quant à « sa propre portée ontologique ». Cette thèse permet à Badiou de préciser son principal point de désaccord avec Lacan en maintenant que la philosophie, au lieu d'être bouchée aux mathématiques, est, au contraire, celle qui, identifiant dans la mathématique « un point de bouchon par rapport à sa propre nature on-

\footnotetext{
$89 \quad$ Ibid.p. 44.

$90 \quad$ Ibid., p. 46.
} 
tologique », tente depuis Platon « de la déboucher ». À cet égard, le philosophe « n'est pas celui qui est bouché aux mathématiques », comme le soutient Lacan, «mais celui qui tente de les déboucher au regard d’elles-mêmes ${ }^{91}$ ».

Badiou s'interroge sur " cette dimension radicale de la mathématique ${ }^{92}$ » que la philosophie est censée manquer ou ne pas saisir. Il trouve la réponse dans "L'étourdit », où Lacan soutient, à propos de la mathématique, justement, que « le dit se renouvelle de prendre sujet d'un dire plutôt que d'aucune réalité93 ». On trouve ce point névralgique dans ce que Lacan propose comme la dialectique du dire et du dit, telle qu'il la développe dans « L'étourdit». Pour Lacan, c'est lorsque « surgit un dire qui ne va pas toujours jusqu'à pouvoir ex-sister au dit », qu' " un certain réel peut être atteint ${ }^{44}$ ». Le surgissement d'un dire dont dépend le dit constitue un événement au sens où il introduit une scansion, une coupure. Mais pour qu'un dire ait lieu, il faut qu'il se soumette à l'épreuve de ses conséquences, du dit, et c'est seulement à ce moment-là qu'un « certain réel peut être atteint ».

Selon la lecture que Badiou propose de cette dialectique singulière du dit et du dire, on peut être d'accord avec Lacan qu'il faut qu'il y ait le dire pour pouvoir transformer, inventer ou renouveler le dit. C'est que la mathématique, en se rapportant au dire, et non pas à une quelconque réalité, se rapporte à une réalité qui lui est immanente, un point sur lequel a déjà insisté Cantor. À suivre Lacan, c'est justement ce point que la philosophie, dans son rapport aux mathématiques, rate forcément, à savoir que le renouvellement du dit s'enracine dans le dire, plutôt que dans le sens comme le prétend la philosophie. En s'efforçant de donner un sens à la vérité, dit Lacan, la philosophie méconnaît la vrai nature de la mathématique qui serait, selon Lacan, mais lu par Badiou, « un dire in-sensé qui se réalise comme dit absolu (intégralement transmissible) ${ }^{95}$ ». Et pour être encore plus précis, «la valeur paradigmatique de la mathématique est d'être le modèle insurpassable d'une pensée qui n'a aucun sens ${ }^{96} »$. Il est curieux d'ailleurs, constate Badiou, que sur cette question, un antiphilosophe, Lacan,

\footnotetext{
91 Ibid., pp. 49-50.

92 Ibid., p. 113.

93 Lacan, «L'étourdit », p. 452.

94 Lacan, Encore, p. 25.

95 Badiou, Lacan, p. 116.

96 Ibid., p. 118.
} 
puisse rejoindre un philosophe, Platon, à une différence essentielle près : ce que Lacan identifie comme valeur paradigmatique de la mathématique est précisément ce que Platon lui reproche.

Car ce que Platon objecte à la mathématique, c'est de fonctionner à partir d'hypothèses dont elle ne rend pas compte. Traduit en termes lacaniens, Platon saisirait, avant Lacan, que la mathématique s'origine dans " un pur dire », que « la mathématique n'est que sous la garantie d'un dire », que Badiou traduit en "dimension axiomatique de la mathématique ${ }^{97}$ ». Vu sous cet angle, le dit, dans la mathématique, "procède intrinsèquement d'un dire, puisqu'il faut que quelque chose soit d'abord dit, pour qu'ensuite il y ait « un enchaînement fidèle à ce dire premier constituant ${ }^{98}$ ». Tout en critiquant la mathématique, Platon rejoindrait Lacan, puisque, lui aussi, identifie « le primat du dire » dont découlerait la réduction du sens. Si la philosophie est le domaine de la dialectique du sens, la mathématique est le domaine de « la prescription du dire » ou " sous la loi de la prescription du dire99 », qui ne se soutient d'aucune garantie de la nécessité. Tirant la leçon de Descartes, Badiou affirme que la spécificité de la discursivité mathématique se fonde sur « l'événement du dire » et ne peut donc être qu'événementielle et contingente, mais, d'un autre côté, « ne relevant pas de la réalité, est absolument nécessaire », à prendre au sens d'être « nécessaire sous l'autorité du dire ${ }^{100}$ ». Ainsi, d'avoir identifié « la mathématique sous la loi du dire », la philosophie doit reconnaître le paradoxe constitutif de la mathématique, le lien entre la contingence et la contrainte ${ }^{101}$.

La lecture que propose Badiou de la «triangulation » : philosophie, psychanalyse, mathématique, n'est peut-être pas aussi claire qu'il le prétend. De fait, il y a plus de points de convergence que de points de divergence entre lui et Lacan que Badiou serait prêt à l'admettre. Voyons de plus près comment fonctionne cette dialectique dans le cas de la mathématique, si la place du dire dans la psychanalyse est « l'analogue dans le discours mathématique ${ }^{102}$ ». En effet, le discours mathématique, si l'on suit Lacan, doit être situé à partir de la logique

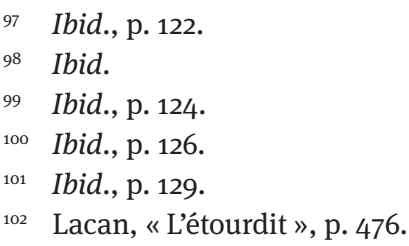


du dire et du dit : «C'est ainsi que le dit ne va pas sans dire. Mais si le dit se pose toujours en vérité, ... le dire ne s'y couple que d'y ex-sister, soit de n'être pas de la dit-mension de la vérité. $»^{103} \mathrm{Si}$ cette logique du dire met en place l'essentiel de la conception lacanienne de la mathématique, c'est aussi parce que, dans le discours de la mathématique, "le dit se renouvelle [constamment] de prendre sujet d'un dire plutôt que d'aucune réalité, quitte, ce dire, à le sommer de la suite proprement logique qu'il implique comme dit $^{104} \gg$.

Pour prendre un exemple, celui d'ailleurs que Lacan lui-même prend, s'il y a un " événement Cantor ", une révolution cantorienne, c'est parce qu'il y a le dire de Cantor ou plutôt l'événement de son dire. Autrement dit, pour Lacan, l'invention mathématique procède du dire, le dire de Cantor, d'Euclide,... puisque seul un dire pourra renouveler le dit défaillant. Or ce dire intervient comme une contingence qui ne se fonde d'aucune réalité. Au départ, le dire n'est que la possibilité de son existence et de ce fait même la suspension de la valeur de vérité du dit. Le dit dépend nécessairement de cette possibilité de l'existence du dire, mais c'est en tant que sa condition que l'acte de dire ex-siste au dit. Étant modale, la logique du dire donne ainsi un nouvel éclairage à la question de l'existence. Pour clarifier ce point, Lacan prend deux exemples, celui du discours psychanalytique et celui du discours mathématique. Concernant le discours psychanalytique, pour qu'il y ait ce discours et sa pratique, l'analyse, il faut restituer « le dire de Freud » qui «s'infère de la logique qui prend de source le dit de l'inconscient ${ }^{105}$ ». Autrement dit, c'est parce que Freud l'a découvert que le dit de l'inconscient existe. L'autre exemple exposé par Lacan, celui du discours de la mathématique, est destiné à montrer que le dit « se renouvelle de prendre sujet d'un dire plutôt que d'aucune réalité ». Ce dire est un acte, dans son pouvoir de transformation, apportant la nouveauté d'une formule mathématique, d'un chiffrage du réel. On peut assigner au dire de Cantor, c'est-à-dire, à un dire qui a déjà fait preuve quant à sa puissance de création d'un nouveau savoir mathématique, le statut de réel, le statut d'ex-sistence au système de savoir en place. Le dit, en revanche, est ce qui, d'être posé en vérité, doit être mis à l'épreuve par la démonstration afin de valider, rétroactivement, le dire

\footnotetext{
${ }^{103}$ Ibid., p. 452.

104 Ibid.

105 Ibid., p. 454.
} 
inaugural. On pourrait dire aussi que le dit issu de ce dire est sommé - via la démonstration - d'assurer au dire son statut axiomatique.

A cet égard, le " dire mathématique », est « le dire » en tant qu'il réalise un savoir. L'avoir lieu du dire présuppose une rencontre inédite, une nouveauté absolue, d'où l'idée de Lacan de faire du "dire » un événement en tant que rencontre avec « un bout de réel ». Ce changement de perspective permet de mettre en place le savoir du réel à partir d'un dire. Pour fixer les termes, on pourrait dire qu'il y a du « dire » au moment où s'établit un avant et un après. L'introduction d'un savoir nouveau en tant que rencontre avec « un bout de réel », produit cet avant et cet après. C'est donc cette rupture ou scansion du temps qui fait du « dire " un événement (mais à condition de ne pas le confondre ni avec le discours mathématique ni avec la démonstration, qui se construisent à partir de ce moment du dire). C'est en ce sens que le « dire-événement » peut conduire à la refonte du champ du savoir dans son ensemble. C'est là, pour reprendre les définitions de Russell, que le mathématicien " ne sait jamais de quoi il parle » parce qu'il confond événement en tant que création pure (en termes badiousiens on dira : la vérité), et savoir en tant qu'ordonnancement rationnel du discours, « ni si ce qu'on dit est vrai » parce qu'il ne fait pas la différence entre les registres qui ordonnent dire et vrai. On voit bien qu'ici, l'affinité entre langage du mathématicien et inconscient se situe au niveau du rapport à la question de la vérité : «le mathématicien a avec son langage le même embarras que nous avec l'inconscient, à le traduire de cette pensée qu'il ne sait pas de quoi il parle, fût-ce à l'assurer d'être vrai (Russell) ${ }^{106}$ ». Dans la perspective de cette affinité, Lacan dit qu'un certain réel est atteint quand « surgit un dire qui ne va pas toujours jusqu'à pouvoir ex-sister au dit ${ }^{107} »$.

\section{Pour conclure}

Sa lecture attentive, mais critique de l'usage lacanien des mathématiques nous permet de voir sous un autre angle la tentative de Badiou de réintriquer philosophie et mathématique. Du fait d'avoir fondé son projet philosophique sur la thèse de l'identité entre mathématique et ontologie, c'est-à-dire d'avoir affirmé que la philosophie est sous la condition des événements de la mathématique, il s'agit chez Badiou d'un tout autre rattachement à la mathématique. En effet, le

\footnotetext{
${ }_{106}$ Ibid., p. 452-453.

107 Lacan, Encore, p. 25.
} 
rattachement de la philosophie consiste en ce qu'elle accepte de reconnaître la mathématique comme l'instigatrice possible d'une détermination inédite de la philosophie elle-même. En tant que « condition » de la philosophie, la mathématique est considérée ici comme puissance d'une pensée libre et laïque dont l'apport est de l'ordre d'une chance de nouveauté donnée. Or, dans cet effort pour relier de nouveau la philosophie et la mathématique, une figure étrange est convoquée : Lacan, qui n'est ni philosophe ni mathématicien, mais psychanalyste. Et c'est dans cette tentative de renouer avec la mathématique, que Badiou critique l'usage de la mathématique par Lacan.

La « triangulation » (mathématique, psychanalyse, philosophie) serait, selon Badiou, une articulation singulière bâtie par Lacan dans le but de mieux circonscrire son recours aux mathématiques. Or, Badiou, lui aussi, a besoin d'une telle " triangulation " pour mettre en lumière la manière dont la philosophie, théorie générale des vérités événementielles, aborde les mathématiques identifiées à la pensée de l'être. Et lui aussi fait référence aux impasses de la formalisation mathématique qui attestent que la mathématique, d'avoir touché à une impossibilité qui lui est propre, se fonde sur un réel. Le réel indépassable de la psychanalyse : il n'y a pas de rapport sexuel ; c'est le réel propre à la psychanalyse en tant que telle, le réel autour duquel tout le « reste » s'ordonne, tout ce qui peut s'inscrire, se mathématiser, formaliser, être transformé en mathèmes. Or, Badiou, n'est-il pas, lui-aussi, obligé de reconnaître qu'il y a un réel ininscriptible de la mathématique cantorienne, le « il n’y a pas de rapport » mesurable, numérable entre le dénombrable et le continu, entre l'ensemble des éléments d'un ensemble et l'ensemble de ses parties, pour s'assurer de la compatibilité entre la philosophie en tant que pensée de l'événementialité des vérités et l'ontologie=mathématiques en tant que pensée de l'être-multiple?

Car dans son effort de renouer les liens entre la philosophie et les mathématiques, la philosophie est forcée de se battre sur plusieurs fronts : contre les philosophies qui ne reconnaissent pas les mathématiques comme une pensée créative (Heidegger, par exemple) ; contre l'antiphilosophie qui assigne les mathématiques à la non-pensée ; et contre l'antiphilosophie singulière de Lacan qui accorde aux mathématiques le statut de pensée parce que les mathématiques, selon Lacan, sont les seules capables de toucher le réel. Mais il ne leur accorde ce privilège qu'au prix de s'arroger le droit d'identifier le point où les mathématiques défaillent : le point d'impossible ou le réel non mathémati- 
sable, ininscriptible en mathèmes, et qui ne peut se manifester qu'à travers les impasses de la formalisation. Car, quelle est l'objection principale que Badiou adresse à Lacan ? De prendre la position que Badiou qualifie d' " archiscientifique » vis-à-vis des mathématiques dans le but d'identifier le point d'impossible indépassable des mathématiques, le « réel du réel » des mathématiques, comme le dit Badiou, plus précisément, en en mot, de vouloir « dire le vrai du réel » des mathématiques. Sortant ainsi du strict cadre des mathématiques, Lacan se hisse à une position que Badiou qualifie d' " archiscentifique », une position qui serait capable de circonscrire le réel non-mathématisable dans les mathématiques elles-mêmes.

Mais en dépit de points de convergence que nous avons essayé de circonscrire, la philosophie badiousienne et la psychanalyse lacanienne n'ont pas et ne peuvent pas avoir le même recours à la mathématique. Pour Lacan, la mathématique, tout en faisant partie de ces disciplines dont elle ne peut pas se désintéresser et tout en admettant qu'elle y occupe une place privilégiée, ne constitue pas une de ses conditions, au sens que Badiou donne à cette notion. Il se tourne vers la mathématique pour garder le cap sur le réel, pour cerner le réel propre à la psychanalyse, tentant en même temps de produire un savoir transmissible à tous via les mathèmes. Cependant la psychanalyse ne dépend pas de la mathématique. D'ailleurs l'invention de la psychanalyse par Freud en témoigne puisque, pour établir la psychanalyse, Freud n’a pas besoin des mathématiques.

Or, tel n'est pas le cas pour Badiou. La philosophie dépend des événements qui ont eu et auront lieu dans la mathématique parce que c'est à partir des événements que de nouvelles vérités se produisent, que la tâche de la philosophie est de penser. La philosophie n'est donc pas et ne peut pas être indifférente à ce qui se passe dans la pensé de l'être en tant qu'être. Ainsi, comme cela a déjà été noté et comme Badiou lui-même le reconnaît, toute mathématique, identifié à l'ontologie, n'est pas compatible avec la philosophie des vérités événementielles. En effet, seule une mathématique capable d'identifier et de penser les points d'impossible, son réel, qui se présente sous la forme de ses impasses, apories, difficultés, fournit la place nécessaire pour que les événements aient lieu. On dira que la philosophie sous la condition dépend non seulement des vérités que ses conditions produisent, mais même plus encore, qu'elle dépend du réel de ses conditions et tout particulièrement du réel de la mathématique $=$ ontologie. 
Badiou lui-même nous donne une idée de cette dépendance de la philosophie du réel de la mathématique. Citons le passage central de l'Être et l'événement :

C’est depuis ses origines que la philosophie, anticipant la butée cantorienne, a scruté l'abîme qui sépare la discrétion numérique du continu géométrique. Cette abîme n'est autre que celui qui sépare $\omega_{\text {o }}$, domaine infini dénombrable des nombres finis, de l'ensemble de ses parties $\mathrm{p}\left(\omega_{0}\right)$, seul apte a fixer la quantité des points dans l'espace. ....Nous pouvons maintenant dire que c'est l'être même, tel que flagrant dans l'impasse de l'ontologie, qui organise l'inexhaustion de sa pensée, dès lors que nulle mesure ne se laisse prendre du lien quantitatif entre une situation et son état, entre l'appartenance et l'inclusion. Il y a tout lieu de croire que c'est pour toujours qu'est ouverte dans l'être cette provocation au concept qu'est le dé-rapport entre présentation et représentation. ... Si le réel est l'impossible, le réel de l'être, soit l'Être, sera précisément ce que détient l'énigme d'un anonymat de la quantitéér.

L'errance de l'être, invoquée par Badiou, est insupportable pour la pensée qui veut, au contraire, que " le désancrage quantitatif de l'être » cesse, et qui donc ne peut se satisfaire de «la dé-mesure ontologiquement attestée», qui vise précisément à mesurer l'excès, à cerner l'être là où « il n'est plus exactement dicible 109 ». Et Badiou examine attentivement trois stratégies de la pensée, trois orientations dans la pensée ontologique, comme il le dit, pour " parer à l'excès ", de dire l'impossible-à-dire de l'ontologie : la voie du constructible, la voie des grands cardinaux et la voie du générique. Si la voie du constructible et la voie des grands cardinaux tentent de maîtriser le réel de l'ontologie, soit « par le bas », c'est-à-dire en réduisant les multiplicités au constructible, soit " par le haut », en formulant des hypothèses d'existences des cardinalités énormes dans le but de prescrire " une disposition hiérarchique où rien ne saurait errer ${ }^{110}$ », l'enjeu de la voie du générique, en revanche, n’est pas de réduire ou de contrôler l'excès, mais de le rejoindre. Pour s'assurer de la compatibilité de la philosophie, théorie générale de l'événementialité des vérités, et de l'ontologie, science de l'être, il ne suffit pas de seulement choisir la voie générique. Il faut en plus anticiper en quelque sorte que la démonstration de l'hypothèse du continu,

\footnotetext{
108 Badiou, Etre et événement, pp. 311-312.

109 Ibid., p. 312.

${ }_{110}$ Ibid., p. 313.
} 
postulant que la cardinalité de $\mathrm{R}$ (le continu ou l'ensemble des nombres réels) est successeur immédiat de celle de $\mathrm{N}$ (dénombrable, ensemble des entiers), ne validera jamais sa vérité. La philosophie, telle que Badiou la conçoit, nécessite une ontologie capable de garantir " pour toujours » qu'il y aurait de nouvelles contingences événementielles dans lesquelles s'originent les vérités, y compris celles de l'ontologie. L'Immanence des vérités semble reprendre et renouveler ce questionnement entamé dans l'Être et l'événement, un questionnement au cœur duquel gît le statut indécidable de l'hypothèse du continu. Badiou opte, comme toujours, pour sa fausseté. La théorie des grands cardinaux, situé dans le camp de l'adversaire d'antan, avec le théorème de Jensen, qui permet de déterminer la puissance d'un ensemble, nommé $0^{\#}$, semble confirmer le bien-fondé du choix badiousien. N'empêche que l'hypothèse que Badiou lui-même fait sur l'hypothèse du continu, à savoir sur sa fausseté foncière, ne se fonde que d'un pari : " un pari sur le réel », qui tout comme le réel de la psychanalyse : il n'y a pas de rapport sexuel, un réel indépassable pour la psychanalyse, constitue l'horizon indépassable pour l'ontologie pour qu'elle puisse être compatible avec la philosophie, pensée des vérités événementielles. Ce que Badiou cherche, c'est un analogue du réel de la psychanalyse, et il le trouve sous une double forme : celle de la fausseté supposée de l'hypothèse du continu, d'une part, et, d'autre part, celle de l'existence supposée d'un ensemble $0^{\#}$.

Dans les deux cas, il s'agit de parier sur l'existence des ensembles infinis non constructibles. Le problème que le "lemme de recouvrement " de Jensen, comme Badiou l'appelle, résout est le suivant : "Il existe un ensemble, nommé $0^{\#}$, zéro dièse, tel que, s'il n'existe pas, le recouvrement est toujours possible, et que, s'il existe, le recouvrement de certaines grandes multiplicités est impossible ${ }^{111}$ ». Or, comme pour l'hypothèse du continu, l'existence de $0^{\#}$ n'est pas démontrable dans ZFC, l'existence de $0^{\#}$ " relève d'une décision », celle qui proclame son existence « au risque même ... que toute l'architecture de l'ontologie formelle s'effondrie ${ }^{112} »$. Or, ce risque ne peut qu'être assumé par le choix qui vise la création ou l'émancipation. Finalement, toute pensée créative, écrit Badiou, est obligée de prendre l'existence de $0^{\#}$ comme axiome, pour pouvoir franchir le cadre finitiste de la situation donnée. Quelle est la leçon à tirer de l'examen minutieux de la théorie des grands cardinaux que présente L'Immanence des

\footnotetext{
111 Badiou, L’immanence des vérités, p. 445.

${ }_{112}$ Ibid., p. 446.
} 
vérités. La réponse que propose Badiou est la suivante : parier sur l'existence de $0^{\#}$ doit être considéré comme une arme pour mener à bien la lutte dans des situations concrètes. Et pour mener à bien cette lutte, il faut

déterminer le seuil, qui est aussi le lieu du combat. Il faut penser l'infini complet - qui nous dit que tout n'est pas soumis à la finitude - mais aussi penser et pratiquer $0^{\#}$ - qui nous dit où se joue la sortie du vieux monde. Et cet « où », infecté qu'il est par les lois du vieux monde et éclairé aussi bien par l'infini nouveau, nul n’en peut économiser les aspects retors, embrouillés, épuisants ${ }^{113}$.

Ainsi, si la mathématique peut créer une possibilité nouvelle pour la philosophie, elle ne le peut que grâce à la contingence de ce que le dire mathématique y produit. La possibilité nouvelle que produit la mathématique n'est donc pas une simple affaire de démonstration, mais implique un acte qui est sans garantie. Nous voici, encore une fois, face à la dialectique du dire et du dit, ou, pour Badiou, face à la dialectique de l'acte et de l'œuvre : tout comme pour Lacan, pour qui c'est à travers l'épreuve du dit que s'assure le statut d'un vrai acte du dire créatif, novateur, pour Badiou, " les actes ne sont rien s'ils ne sont eux aussi des œuvres ${ }^{114} »$.

\section{Références}

Badiou, Alain, Théorie du sujet, Seuil, Paris 1982

- L'Être et l'événement, Seuil, Paris 1988

- «Philosophie et mathématique », Conditions, Seuil, Paris 1992

- «Sujet et infini », Conditions

- « La mathématique est une pensée », Court traité d'ontologie transitoire, Seuil Paris 1998

- Le Siècle, Seuil, Paris 2005

- Le Séminaire. Lacan. L'antiphilosophie 3, 1994-1995, Fayard, Paris 2013

- Éloge des mathématiques, Flammarion, Paris 2015

- L'immanence des vérités, Fayard, Paris 2018

Cantor, Georg, Gesammelte Abhandlungen mathématischen und philosophischen Inhalts, E. Zermelo (éd.), Springer, Berin 1932

\footnotetext{
113 Ibid., p. 462.
}

114 Ibid. 
- «Fondements d'une théorie générale des ensembles », trad. J.-C. Milner, Cahiers pour l'analyse (10/1969)

Charraud, Natalie, Infini et Inconscient. Essai sur Georg Cantor, Anthropos-Economica, Paris 1994

Imbert, Claude, "Où finit le platonisme ? ", dans Alain Badiou : Penser le multiple, Charles Ramond (éd.), L'Harmattan, Paris 2002

Kant, Emmanuel, Critique de la raison pure, dans Euvres philosophiques, tome I : Des premiers écrits à la Critique de la raison pure (1747-1781), Bibliothèque de la Pléiade, Gallimard, Paris 1980

Lacan, Jacques, «Science et vérité », dans Écrits, Seuil, Paris 1966

- «Séminaire de Jacques Lacan, Le savoir du psychanalyste, 1971-1972 » (inédit)

- «Séminaire de Jacques Lacan, XXII, R.S.I. 1972-1973 », (inédit)

- Le Séminaire de Jacques Lacan, Livre XX, Encore, Seuil, Paris 1975

- «Séminaire de Jacques Lacan, XXV, Le moment de conclure 1977-1978 », (inédit)

- Le Séminaire de Jacques Lacan, Livre XVII, L'envers de la psychanalyse, Seuil, Paris 1991

- «L'étourdit », dans Autres écrits, Seuil, Paris 2001

- «La note italienne », Autres écrits

- « Télévision », Autres écrits

Lavendhomme, René, Lieu du sujet. Psychanalyse et mathématique, Seuil, Paris 2001

Miller, Jacques-Alain, «Vers un signifiant nouveau », Revue de l'ECF (20/1992)

Russel, Bernard, Écrits de logique philosophique, trad. Jean-Michel Roy, PUF, Paris 1989

- « Work on the principles of mathematics », The International Monthly, 4 (1/1901) 